\title{
Infant Midnasal Stenosis: Reliability of Nasal Metrics
}

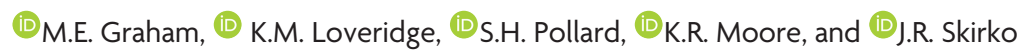

\begin{abstract}
BACKGROUND AND PURPOSE: Midnasal stenosis is a poorly defined entity that may be a component of other conditions of nasal obstruction contributing to respiratory distress in infants. We sought to establish whether midnasal vault narrowing is a component of well-defined syndromes of nasal narrowing, such as bilateral choanal atresia and pyriform aperture stenosis, and to characterize the nasal anatomy of patients with syndromic craniosynostosis.
\end{abstract}

MATERIALS AND METHODS: A convenience sample of patients with pyriform aperture stenosis, bilateral choanal atresia, and Apert and Crouzon syndromes with maxillofacial CT scans was identified. Patients with Pierre Robin Sequence were used as controls. Nasal measurements were performed at the pyriform aperture, choana, and defined midnasal points on axial and coronal CT scans. Intra- and interrater reliability was quantified with the intraclass correlation coefficient. $T$ tests with Bonferroni adjustment were used to assess differences from controls.

RESULTS: The study included 50 patients: Eleven had pyriform aperture stenosis, 10 had Apert and Crouzon syndromes, 9 had choanal atresia, and 20 were controls. Measurements in patients with pyriform aperture stenosis and Apert and Crouzon syndromes were narrower than those of controls at all measured points $(P<.001)$. Measurements in patients with choanal atresia were only narrow in the posterior half of the nose $(P<.001)$. The intra- and interrater reliability of midnasal and pyriform measurements was very good to excellent (intraclass correlation coefficient $>0.87$ ). The choanal measurement was good (intraclass correlation coefficient $=0.76-0.77$ ).

CONCLUSIONS: Pyriform aperture stenosis, Apert and Crouzon patients were narrower at all measured points compared to controls. Bilateral choanal atresia patients were only narrower in the posterior half of the nose. More research is needed to evaluate the clinical implications of these radiographic findings.

ABBREVIATIONS: $B C A=$ bilateral choanal atresia; $\mathrm{ICC}=$ intraclass correlation coefficient; $\mathrm{LD}=$ lacrimal duct; $\mathrm{LM}=$ last molar; $\mathrm{PAS}=$ pyriform aperture stenosis; PRS = Pierre Robin Sequence; $S C=$ syndromic craniosynostosis (includes Apert and Crouzon syndromes)

$\mathbf{N}$ eonates are obligate nasal breathers. Stenosis at the pyriform aperture (pyriform aperture stenosis [PAS]) or the choana (bilateral choanal atresia $[\mathrm{BCA}]$ ) may lead to severe nasal obstruction, necessitating intubation and/or surgical intervention in infants. Each condition is relatively rare, with an incidence of pyri-

Received August 13, 2018; accepted after revision January 14, 2019.

From the Department of Otolaryngology-Head and Neck Surgery, and Schulich School of Medicine and Dentistry (M.E.G.), Western University, London, Ontario Canada; London Health Sciences Center (M.E.G.), London, Ontario, Canada; Division of Pediatric Otolaryngology-Head and Neck Surgery (K.M.L., S.H.P., J.R.S.), University of Utah and Primary Children's Hospital, Salt Lake City, Utah; and Department of Medical Imaging (K.R.M.), Primary Children's Hospital, Salt Lake City, Utah.

Paper previously presented at: Annual Meeting of the American Society of Pediatric Otolaryngology, April 18-22, 2018; National Harbor, Maryland,

Please address correspondence to Jonathan R. Skirko, MD, MHPA, MPH, Division of Pediatric Otolaryngology, University of Utah and Primary Children's Hospital, $100 \mathrm{~N}$ Mario Capecchi Dr, Salt Lake City, UT 84113; e-mail: jonathan.skirko@hsc.utah.edu; (®)_SkirkoMD

http://dx.doi.org/10.3174/ajnr.A5980 form aperture stenosis of 1 in 25,000 live births ${ }^{1}$ and choanal atresia overall occurring in 1 in 5000-8000 live births, most commonly unilateral. ${ }^{2}$ Children with syndromic craniosynostosis (SC), such as Apert and Crouzon syndromes, likewise often require management of nasal obstruction as a part of their multidisciplinary care. In one series examining the airway manifestations in Apert syndrome, $60 \%$ of patients were found to have CT evidence of nasal abnormalities, including BCA, bilateral or unilateral bony nasal stenosis, and septal deviation. ${ }^{3}$

When PAS or BCA causes anatomic obstruction, the clinical and radiographic diagnosis is relatively straightforward and measurements at these specific sites have been previously described. ${ }^{4,5}$ Reeves et $\mathrm{al}^{6}$ characterized the dimensions of the bony pyriform aperture and the more posterior nasal cavity in the imaging of patients with PAS and found that there was associated posterior narrowing. In most other studies, the remainder of the nasal cavity was not discussed in evaluating patients with BCA and 

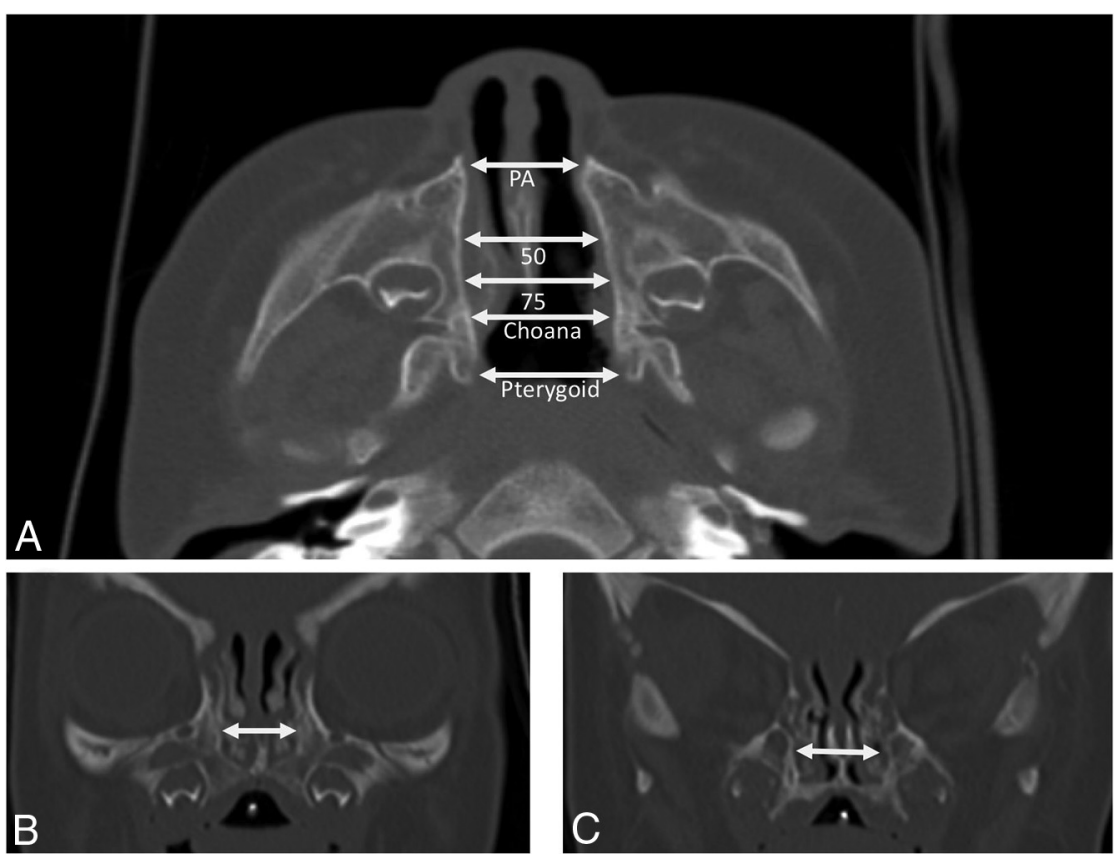

FIG 1. Measurement landmarks. Axial scans $(A)$ were measured from bony lateral nasal wall to bony lateral nasal wall at the level of the pyriform aperture (PA), the choana, and at points $50 \%$ and $75 \%$ posteriorly between these two landmarks. An additional measurement on axial scan was obtained between the medial pterygoid plates. On coronal views, measurements were obtained from bony lateral nasal wall to bony lateral nasal wall at the pyriform aperture (not shown), just posterior to the lacrimal duct (B), and at the last molar (C).

PAS. ${ }^{1,2,4}$ Previous work on Crouzon syndrome identified the nasal cavity as the narrowest portion of the airway by fluid dynamics and 3D modeling but did not quantify nasal dimensions on CT, which is more easily used in clinical practice. ${ }^{7} \mathrm{We}$ are not aware of any studies quantifying the nasal caliber in SC in this way.

Providing comparisons of nasal metrics across nasal-obstruction disorders and controls is the first step in improving our understanding of the nasal airway beyond complete or near-complete obstruction at the pyriform aperture and choana. Given the ability to measure the nasal airway at many different points and in different planes on imaging, the reliability of measurements may also help decide which measurements would be the most useful moving forward. Although abnormalities are suspected from previous work, the degree of midnasal narrowing, if present, is not definitively known in patients with BCA and PAS. ${ }^{2,6}$ While it is also suspected that SC may be associated with midnasal or transnasal stenosis, this association has not been established or quantified.

We sought to determine the area or areas of stenosis in patients with SC, the degree of stenosis, and the caliber of the remainder of the nasal cavity in PAS/BCA in comparison with a control group of patients. Additionally, we sought to test the reliability of our measurements to define nasal stenosis from both the axial and coronal planes of the CT scan.

\section{MATERIALS AND METHODS \\ Population}

A retrospective convenience sample of patients with CT scans of the maxillofacial skeleton meeting 1 of the 4 categories of diagnoses was used. Diagnoses included PAS, BCA, Apert or Crouzon syndrome, or Pierre Robin Sequence ([PRS] used as the control group), confirmed by chart review. Inclusion criteria required an available maxillofacial CT scan before 3 months of age with coronal and axial slices through the nasal floor. Patients with Apert and Crouzon syndromes were grouped together as having "syndromic craniosynostosis" because these groups were each small and have a similar etiology and facial morphology. PRS was used as a control group because these children are not known to have associated nasal narrowing and represent a uniform population of infants with a maxillofacial CT scan in early infancy.

\section{Radiographic Measurements}

CT measurements were performed in the axial view for each patient as has been described in the literature previously, ${ }^{6,8}$ with the caliper tool in standard imaging software (Impax; Agfa-Gevaert, Mortsel, Belgium). Axial measurements were performed using the following landmarks: pyriform aperture, choana, and points $50 \%$ and $75 \%$ between these 2 landmarks, and between the medial pterygoid plates (millimeters) (Fig 1A). Raters scrolled through the images to find the identified landmarks independent of one another in each patient.

In the coronal view, 3 landmarks were used. The pyriform aperture width was measured from the first frame in which it was fully visible, slightly above the floor of the nasal cavity, immediately below the head of the inferior turbinate. The nasal width posterior to the lacrimal duct (measurement [LD]) was determined by taking a measurement on the scan directly posterior to the last frame in which the lacrimal duct could be identified (Fig $1 B)$. The width of the posterior nasal cavity was measured in a similar manner at the level of the last molar (measurement [LM]) (Fig 1C).

Distances were independently measured at the above points by 2 raters (J.R.S., K.M.L.). Each rater performed all measurements a second time 1 week after first data collection. The raters were not formally blinded to the diagnosis because, in most cases, anomalies could be easily diagnosed on reviewing the imaging before performing measurements.

\section{Statistical Analysis}

The groups were compared for differences in age at scanning, which could contribute to differences in measurements across groups. The discriminant validity of the metric tested the ability to detect a difference among groups of nasal disorders. ${ }^{9}$ The nasal dimensions of each condition were compared with those of the control group using a $T$ test. The Bonferroni correction for multiple comparisons was used when defining statistical significance. With 32 


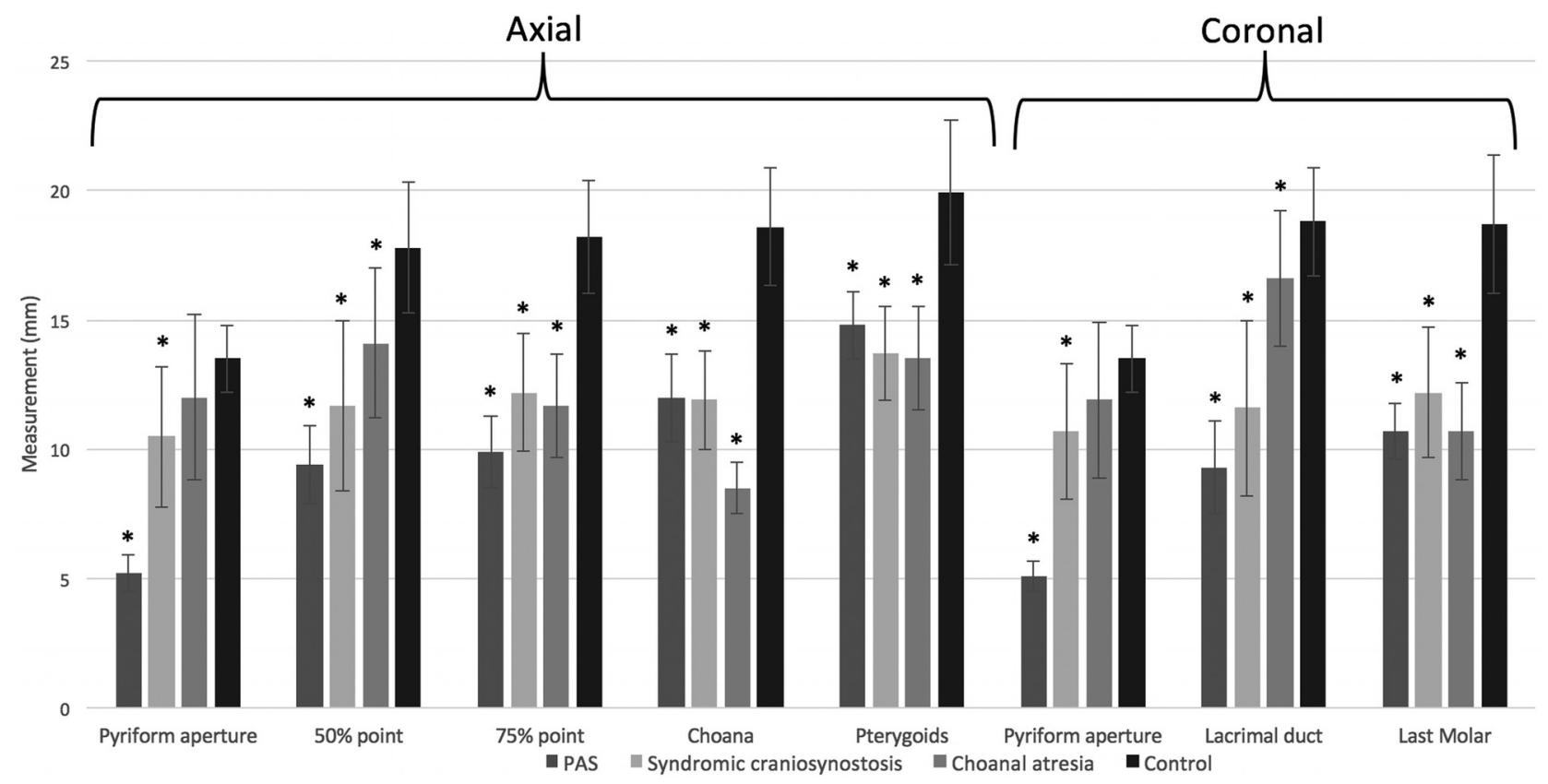

FIG 2. Mean nasal measurements. Measurements are given in millimeters. Asterisks indicate statistical significance $(P<.001)$.

individual tests, the threshold for statistical significance was $P<$ 0.002 .

Intrarater reliability used the intraclass correlation coefficient (ICC) with a $95 \%$ confidence interval to calculate the reproducibility of repeat measurements in the same rater. Interrater reliability was similarly calculated for the reproducibility of repeat measurements in different raters. In a modification of the Altman thresholds for reliability, ${ }^{10}$ values from 0.41 to 0.60 were considered "moderate"; those from 0.61 to 0.80 , "good"; those from 0.81 to 0.90 , "very good"; and those of $>0.90$, "excellent" reliability. Additionally, the difference between the 2 raters' mean measurements at each site was provided in millimeters.

All analyses were performed with STATA/SE 10.1 (StataCorp, College Station, Texas).

\section{RESULTS}

\section{Patient Demographics}

Fifty patients were identified and categorized as having congenital PAS $(n=11)$, SC $(n=9)$, and BCA $(n=10)$ or were controls (PRS) $(n=20)$. Patients were, an average, 0.7 months of age at the time of their scan. Mean ages by diagnostic group were as follows: patients with PAS, $0.50 \pm 0.5$ months; patients with SC, $0.55 \pm 1.6$ months; patients with $\mathrm{BCA}, 0.33 \pm 0.5$ months; and controls, $0.81 \pm 1.1$ months. There was no statistically significant difference in age at scanning between any group and the control group ( $P \geq .2$ on all tests).

\section{Pyriform Aperture}

The mean pyriform aperture measurement on axial slices for patients with PAS was $5.2 \pm 0.7 \mathrm{~mm}$ (Fig 2). Patients with SC had a mean pyriform aperture width of $10.5 \pm 2.7 \mathrm{~mm}$. Patients with BCA had a mean pyriform aperture width of $12.0 \pm 3.2 \mathrm{~mm}$. Controls had a mean pyriform width of $13.6 \pm 1.3 \mathrm{~mm}$. The coronal measurements were similar.

Pyriform aperture measurements in patients with both PAS and SC were narrower than those in controls $(P<.001)$. In comparing patients with BCA with controls, the difference in pyriform aperture dimension was not statistically significant when measured in the coronal $(P=.02)$ or axial $(P=.07)$ plane.

\section{Midnasal Passage}

Midnasal width was measured at 2 sites in both the axial and coronal views (LD and LM on coronal, $50 \%$ and $75 \%$ on axial). Infants with PAS had mean LD and LM measurements of $9.3 \pm$ $1.8 \mathrm{~mm}$ and $10.7 \pm 1.1 \mathrm{~mm}$, respectively. These were narrower than those in controls (PRS) (LD and LM measurements, $18.8 \pm 2.1 \mathrm{~mm}$ and $18.7 \pm 2.7 \mathrm{~mm}$, respectively; $P<.001)$. PAS mean axial measurements were similar to these. Measurements of patients with syndromic craniosynostosis were $11.6 \pm 3.4$ $\mathrm{mm}(\mathrm{LD})$ and $12.2 \pm 2.5 \mathrm{~mm}(\mathrm{LM})$, with similar findings on axial measurements, also narrower than those in controls $(P<$ $.001)$.

In our BCA group, mean coronal measurements were $16.6 \pm$ $2.6 \mathrm{~mm}$ (LD), which were not considered different from those in the controls $(18.8 \mathrm{~mm})$ after adjusting for multiple testing $(P=$ $.03)$. The means for the LM measurement (11.7 $\pm 3.3 \mathrm{~mm}), 50 \%$ measurement $(14.1 \pm 2.9 \mathrm{~mm})$, and $75 \%$ measurement $(11.7 \pm$ $2.0 \mathrm{~mm})$ were all narrower than those in controls $(P<.001)$.

\section{Choana}

The mean choanal measurements were $12.0 \pm 1.7 \mathrm{~mm}$ (PAS), $11.9 \pm 1.9 \mathrm{~mm}$ (SC), and $8.5 \pm 1.0 \mathrm{~mm}$ (BCA). The control mean choanal measurement was $18.6 \pm 2.3 \mathrm{~mm}$. For all 3 conditions, the narrower choanal measurement was statistically significant $(P<.001)$.

\section{Pterygoid}

Patients with PAS, SC, and BCA had mean measurements of $14.8 \pm 1.3 \mathrm{~mm}, 13.7 \pm 1.8 \mathrm{~mm}$, and $13.5 \pm 2.0 \mathrm{~mm}$, respectively, at the level of the pterygoid on axial CT, with the control PRS 
Inter- and intrarater reliability

\begin{tabular}{|c|c|c|c|c|c|c|c|c|}
\hline \multirow{2}{*}{ Coron } & \multicolumn{4}{|c|}{ Intrarater Reliability } & \multicolumn{4}{|c|}{ Interrater Reliability } \\
\hline & ICC & SE & \multicolumn{2}{|c|}{$95 \% \mathrm{Cl}$} & ICC & SE & \multicolumn{2}{|c|}{$95 \% \mathrm{Cl}$} \\
\hline PA & 0.97 & 0.01 & 0.96 & 0.99 & 0.97 & 0.01 & 0.95 & 0.99 \\
\hline LD & 0.94 & 0.02 & 0.89 & 0.98 & 0.94 & 0.02 & 0.90 & 0.98 \\
\hline LM & 0.88 & 0.04 & 0.79 & 0.96 & 0.95 & 0.02 & 0.92 & 0.98 \\
\hline \multicolumn{9}{|l|}{ Axial } \\
\hline PA & 0.97 & 0.01 & 0.95 & 0.99 & 0.96 & 0.01 & 0.94 & 0.99 \\
\hline $50 \%$ & 0.90 & 0.04 & 0.83 & 0.97 & 0.93 & 0.03 & 0.88 & 0.99 \\
\hline $75 \%$ & 0.87 & 0.05 & 0.78 & 0.96 & 0.93 & 0.03 & 0.86 & 0.99 \\
\hline $\mathrm{CA}$ & 0.76 & 0.08 & 0.60 & 0.91 & 0.77 & 0.09 & 0.59 & 0.96 \\
\hline Pty & 0.86 & 0.05 & 0.76 & 0.95 & 0.45 & 0.21 & 0.04 & 0.86 \\
\hline
\end{tabular}

Note:-SE indicates standard error; CA, choana; Pty, pterygoid plate; PA, pyriform aperture.
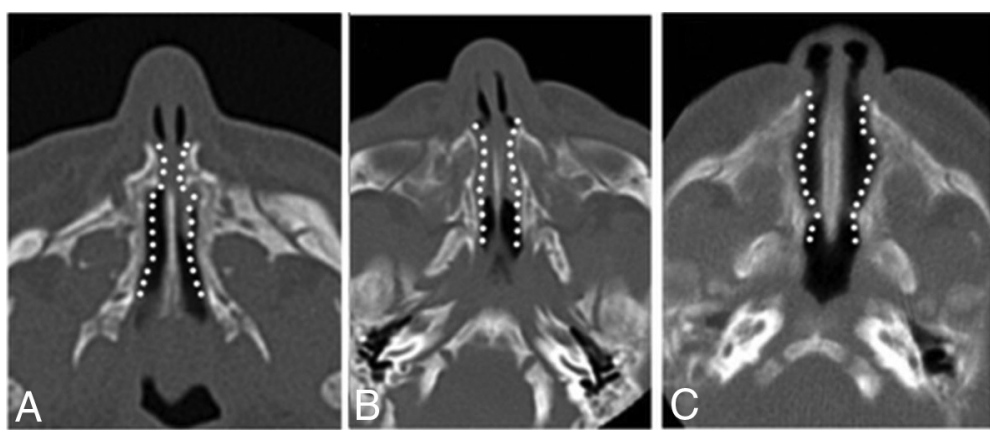

FIG 3. Trends seen in the nasal cavity of pyriform aperture stenosis $(A)$, syndromic craniosynostosis $(B)$, and bilateral choanal atresia $(C)$. Note the more global narrowing in patients with PAS and $\mathrm{SC}$ versus a more posterior narrowing in patients with BCA. Although the pyriform aperture in patients with BCA appears narrower than that in controls (Fig $1 A$ ) visually, this difference is not statistically significant.
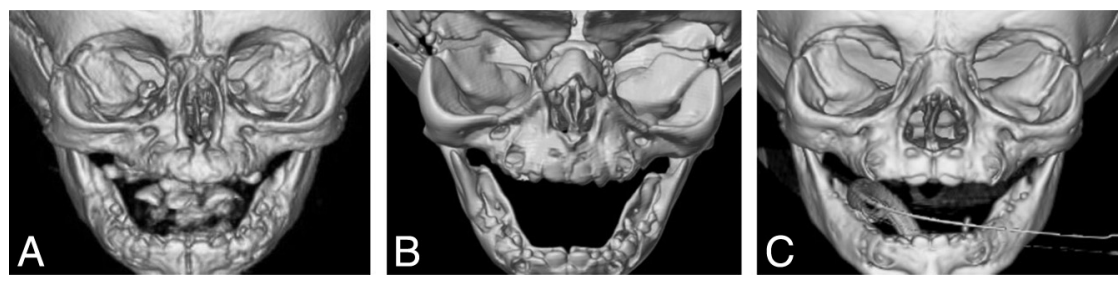

FIG 4. $3 D$ reconstructions of maxillofacial $C T$ scans highlighting the pyriform aperture, in particular in patients with PAS $(A)$, Apert syndrome $(B)$, and PRS (C).

population having a mean pterygoid measurement of $19.9 \pm 2.8$ mm. The mean pterygoid measurement of each group was significantly narrower than that of controls $(P<.001)$.

\section{Intra- and Interrater Reliability}

Intrarater reliability was found to be very good to excellent for all measurements except the choana on axial views $($ ICC $=0.77$, good). Interrater reliability for the medial pterygoid plate was moderate $(\mathrm{ICC}=0.45)$. The remainder of the nasal metrics were excellent, except for the choana (ICC $=0.77$, good), the only other measurement $<0.9$. (Table). The measured difference in millimeters varied by ICC. Those measurements with ICC values of $>0.93$ had a $<1-\mathrm{mm}$ difference in measurements between raters (differences: coronal pyriform aperture $=0.1$ $\mathrm{mm}, \mathrm{LD}=0.8 \mathrm{~mm}, \mathrm{LM}=0.2 \mathrm{~mm}$, axial pyriform aperture $=$ $0.6 \mathrm{~mm}$ ). Those with an ICC $\leq 0.93$ had an average of a 2.2-mm difference between raters (differences: axial 50\% $=2.6$ $\mathrm{mm}$, axial $75 \%=2.7 \mathrm{~mm}$, axial choana $=1.4 \mathrm{~mm}$, axial pterygoid $=2.2 \mathrm{~mm}$ ).

\section{DISCUSSION}

Neonates are obligate nasal breathers, and understanding infant nasal anatomy is therefore very important. ${ }^{11} \mathrm{Nev}$ ertheless, measurements have not been standardized, and in many conditions, only 1 anatomic site is measured. Reeves et $\mathrm{al}^{6}$ described a method of measuring the posterior nasal cavity using the choana and pyriform aperture as initial landmarks on axial CT as well as points $50 \%$ and $75 \%$ between the two. We adapted this technique with the addition of several other points and coronal landmarks. The 8 sites we present have interrater and intrarater reliability that is, at minimum, rated as good, with most being excellent. An exception is in the use of the medial pterygoid plate. It is possible that due to the superoinferior flaring of the plates, the axial plane is not ideal for measurement of this landmark. We suspect that this landmark might have differences among our patient groups as an important buttress of the midface and that narrowing in this location may represent narrowing of the entire maxilla, but the clinical significance of this difference is unclear because it is outside the nasal cavity.

Using our measurement technique, we found that the shape of the nasal passage may be different in the groups with nasal airway obstruction. We found that patients with syndromic craniosynostosis have global narrowing, as seen in the previous fluid dynamic study and in keeping with the symptoms noted in case series. ${ }^{3,7}$ Most interesting, patients with PAS and BCA also appear to have more than an isolated narrowing. Figure 3 illustrates the shape of the nasal cavity on axial scans in each condition. It appears that the nasal cavity of patients with PAS and SC are globally narrow. Patients with BCA also have a narrower nasal passage than controls, but this is only statistically significant in the more posterior nasal cavity, from the $50 \%$ point posterior on axial scans and from the last molar posterior on the coronal cuts. The difference in the pyriform aperture is further illustrated in 3D reconstructions generated from maxillofacial CTs (Fig 4).

Reeves et $\mathrm{al}^{6}$ examined CT scans in patients with PAS and found that the anterior $75 \%$ of the nasal cavity was narrower than that in controls. Their patients with PAS $(n=7)$ had narrower choanae than controls $(n=13)$, but this finding was not statistically significant. In our slightly larger case series of patients with PAS $(n=11)$ and controls $(n=20)$, we found that the nasal cavity was globally narrower, including the choana, and this was statistically significant throughout. Aslan et $\mathrm{al}^{2}$ similarly performed 
detailed analysis of the nasal caliber in patients with BCA. Their measurement sites were different from ours, making comparison difficult, and they did not include a midnasal bony measurement. In contrast to our study, the authors did find a statistically significant difference at the bony pyriform aperture between patients with BCA and controls. Our study used the Bonferroni correction for multiple testing, which might make it less likely that the null hypothesis would be refuted by chance. More studies with a larger sample size and with limited numbers of statistical tests looking more specifically at this site in patients with BCA may reveal whether there is a true global narrowing in patients with BCA as well.

There are few studies of management of the nasal airway in Apert and Crouzon syndromes. A case series in airway management in Apert syndrome indicated that those patients with bony nasal stenosis required an average of 3-5 dilations per patient. ${ }^{3}$ Further defining the problem of narrowing throughout the nasal passage on preoperative imaging may help optimize future treatments for nasal airway obstruction in infants with syndromic craniosynostosis.

The most important clinical implication of our results may be in predicting the likelihood of surgical success. A globally narrow nose as seen in SC and PAS is unlikely to respond to an anatomically directed single-site drilling, and even those patients with BCA have narrowing not limited to the choana. Revision rates in choanal atresia are estimated to be between $6 \%$ and $36 \%{ }^{12,13}$ Because the incidence of PAS is low, there are limited data regarding revision surgery rates. A systematic review and case series from 1 institution included a total of 73 patients undergoing surgical management for PAS, with a $15 \%$ revision rate. ${ }^{14}$ It is possible that surgical failures in these conditions may be due to concomitant stenosis elsewhere that is neither recognized nor managed, but this requires more study.

Our control group (patients with PRS) could be considered a limitation. It is not common for an infant with no known congenital anomalies or syndromes to undergo a maxillofacial CT. Patients with Pierre Robin Sequence commonly undergo CT for surgical planning for mandibular distraction and therefore provide a pool of similarly aged patients, presumably without nasal abnormalities. It is possible, though not described, that they have a wider or narrower nasal passage than nonsyndromic infants, over- or underestimating the difference in nasal dimensions. We did not identify other similarly aged groups undergoing scans; those presenting with peritonsillar or other deep space neck abscesses tended to be older than 5 years of age, and those undergoing CT for cochlear implantation typically are older than 6 months of age. ${ }^{15}$

An additional limitation may be a selection bias in those patients undergoing CT. Patients with milder nasal obstruction, particularly those with pyriform aperture stenosis or choanal atresia may not undergo imaging, and our measurement data might be biased toward patients with narrower nasal cavities. While this bias may be present, it would only limit the generalizability of these results to those patients with less severe nasal obstruction (those who are less likely to be surgical candidates). Nasal metrics in these patients would be unlikely to change management. This limitation is likely not significant in those patients with craniosynostosis who are being imaged for their cranial vault issues rather than nasal obstruction, and we would expect many patients in this group to be included. Some patients with SC had only a head CT, which did not capture the maxillofacial skeleton, limiting our sample size. In patients with SC with Apert syndrome, an additional concern is the potential for orbital asymmetry. ${ }^{16}$ This did not affect the interrater reliability of the LD measurement $(0.97 ; 95 \% \mathrm{CI}, 0.93-1.0)$ but may mean that this measurement represents a different portion of the nose in patients with SC. Future studies of the position of the lacrimal system in these patients may be helpful.

Future directions include analysis of the clinical implications of midnasal narrowing with the goal of defining the best location for evaluating midnasal stenosis and creating a cutoff value for significant stenosis under which poorer outcomes or increased revision rates are more likely. Measuring more control patients would also better define a metric of typical nasal diameter to be used for comparison in future work.

\section{CONCLUSIONS}

There is global narrowing of the nasal airway in patients with PAS and Apert and Crouzon syndromes and narrowing of the mid-toposterior nasal cavity in patients with BCA. This finding highlights the need to evaluate the entire nasal airway in surgical planning and may have implications for the likelihood of surgical success. The anatomic sites we have proposed to evaluate the nasal caliber have good-to-excellent inter- and intrarater reliability and may be extended to use in other studies.

\section{REFERENCES}

1. Wormald R, Hinton-Bayre A, Bumbak P, et al. Congenital nasal pyriform aperture stenosis $5.7 \mathrm{~mm}$ or less is associated with surgical intervention: a pooled case series. Int J Pediatr Otorhinolaryngol 2015;79:1802-05 CrossRef Medline

2. Aslan S, Yilmazer C, Yildirim T, et al. Comparison of nasal region dimensions in bilateral choanal atresia patients and normal controls: a computed tomographic analysis with clinical implications. Int J Pediatr Otorhinolaryngol 2009;73:329-35 CrossRef Medline

3. Xie C, De S, Selby A. Management of the airway in Apert syndrome. J Craniofac Surg 2016;27:137-41 CrossRef Medline

4. Moreddu E, Le Treut-Gay C, Triglia JM, et al. Congenital nasal pyriform aperture stenosis: elaboration of a management algorithm from 25 years of experience. Int J Pediatr Otorhinolaryngol 2016;83: 7-11 CrossRef Medline

5. Wine TM, Dedhia K, Chi DH. Congenital nasal pyriform aperture stenosis: is there a role for nasal dilation? JAMA Otolaryngol Head Neck Surg 2014;140:352-56 CrossRef Medline

6. Reeves TD, Discolo CM, White DR. Nasal cavity dimensions in congenital pyriform aperture stenosis. Int J Pediatr Otorhinolaryngol 2013;77:1830-32 CrossRef Medline

7. Wang T, Mu X, Deng J, et al. Investigation on the structure of nasal cavity and its airflow field in Crouzon syndrome. J Craniofac Surg 2011;22:166-72 CrossRef Medline

8. Belden CJ, Mancuso AA, Schmalfuss IM. CT features of congenital nasal piriform aperture stenosis: initial experience. Radiology 1999; 213:495-501 CrossRef Medline

9. Westen D, Rosenthal R. Quantifying construct validity: two simple measures. J Pers Soc Psychol 2003;84:608-18 CrossRef Medline

10. Altman DG. Practical Statistics for Medical Research. London: Chapman and Hall; 1991

11. Miller MJ, Martin RJ, Carlo WA, et al. Oral breathing in newborn infants. J Pediatr 1985;107:465-69 CrossRef Medline

12. Tatar ȨC, Öcal B, Doğan E, et al. Stentless endoscopic repair of con- 
genital choanal atresia: is it enough for maintaining choanal patency? Eur Arch Otorhinolaryngol 2017;274:3673-78 CrossRef Medline

13. Brihaye P, Delpierre I, De Villé A, et al. Comprehensive management of congenital choanal atresia. Int J Pediatr Otorhinolaryngol 2017;98: 9-18 CrossRef Medline

14. Gonik NJ, Cheng J, Lesser M, et al. Patient selection in congenital pyriform aperture stenosis repair: 14 year experience and system- atic review of literature. Int J Pediatr Otorhinolaryngol 2015;79: 235-39 CrossRef Medline

15. Novis SJ, Pritchett CV, Thorne MC, et al. Pediatric deep space neck infections in U.S. children, 2000-2009. Int J Pediatr Otorhinolaryngol 2014;78:832-36 CrossRef Medline

16. Kreiborg S, Cohen MM Jr. Ocular manifestations of Apert and Crouzon syndromes: qualitative and quantitative findings. $J$ Craniofac Surg 2010;21:1354-57 CrossRef Medline 